\title{
Arginine methylation as a key post-translational modification in skeletal muscle homeostasis: a review
}

\author{
Hyebeen Kim, Jong-Sun Kang, Hyun-Ju Jeong
}

Department of Molecular Cell Biology, Single Cell Network Research Center, Sungkyunkwan University School of Medicine, Suwon, Korea

Received: August 28, 2019

Revised: October 8, 2019

Accepted: October 14, 2019

Corresponding author:

Hyun-Ju Jeong

Department of Molecular

Cell Biology, Sungkyunkwan

University School of Medicine,

2066 Seobu-ro, Jangan-gu,

Suwon 16419, Korea

Tel: +82-31-299-6157

E-mail: nabiya1127@skku.edu

\begin{abstract}
Arginine methylation mediated by protein arginine methyltransferases (PRMTs) has emerged as a key post-translational modification of histone or nonhistone substrates. It involves or controls signaling pathways or gene expression implicated in diverse processes, including muscle regeneration and metabolic homeostasis. Reciprocally, loss of skeletal muscle mass and function related to aging or other pathological conditions could be related to the secondary chronic diseases, for example, metabolic syndromes, chronic inflammation, or cardiovascular diseases. Thus, understanding the pathways that regulate muscle homeostasis is critical to develop therapeutic strategies for preventing muscle loss and related secondary chronic diseases. Recent in vivo research using gene-targeting mouse models have advanced our knowledge about the role of several PRMTs in muscle regeneration and metabolic controls. In this review, we will focus on the recent discoveries on the in vivo function of PRMTs in muscle homeostasis.
\end{abstract}

Keywords: Muscle, skeletal; Regeneration; Metabolism; Protein arginine methyltransferase
This is an Open Access article distributed under the terms of the Creative Commons Attribution Non-Commercial License (http:// creativecommons.org/licenses/ by-nc/4.0/).

\section{INTRODUCTION}

Skeletal muscle is the largest organ in humans, constituting about $40 \%$ of body weight in an adult, and plays critical roles in locomotion, energy expenditure, and glucose disposal [1-3]. Thus, alterations in metabolic characteristics as well as mass or strength of skeletal muscle affect the whole body's energy metabolism and health [4]. Skeletal muscle from healthy adults displays a resilient adaptation in energy metabolism and contractile functions in response to demands like exercise, hormones, or nutritional states. Impairments in muscle metabolism and function have been implicated in metabolic pathologies, such as insulin resistance, glucose intolerance, and obesity $[2,5,6]$.

Aging is accompanied by a progressive loss of muscle mass and function known as sarcopenia, which contributes to the reduced functional capacity and an increased risk for developing secondary chronic diseases, such as metabolic diseases, chronic inflammation, or cardiovas- 
cular diseases [2,7-9]. Thus, research efforts are focused on understanding the underlying mechanisms of muscle aging and to develop strategies to prevent muscle loss and weakness. One of the key factors contributing to muscle aging seems to be related to decreased muscle regenerative capacity accompanied by increased fibrosis. Thus, understanding the regulatory mechanisms of muscle stem cell function is critical for preventing muscle dysfunction.

Post-translational modifications such as phosphorylation, ubiquitination, and lysine methylation of diverse regulators have been shown to play critical roles in a variety of biological processes, including muscle regeneration and metabolic homeostasis [10]. Arginine methylation is a newly emerging post-translational modification of histone or nonhistone proteins. Protein arginine methyltransferases (PRMTs) catalyze mono-methylation and symmetric or asymmetric dimethylation of arginine residues on both histone and non-histone substrates, thereby modulating diverse signaling pathways and expression of genes involved in diverse biological processes, including muscle regeneration and muscle metabolism control [11-13]. PRMTs can be classified as type I, which catalyze asymmetric arginine dimethylation (PRMT1, PRMT2, PRMT3, PRMT4, PRMT6, and PRMT8), type II, which generate symmetric dimethyl-arginine residues (PRMT5 and PRMT9), or type III, which generate monomethyl-arginine residues (PRMT7) [11, 12]. Several recent publications have advanced our understanding about the importance of several PRMTs in the control of muscle regeneration and function. This review focuses on the recent advances regarding PRMT function in controlling muscle stem cell function and controlling muscle metabolism.

\section{THE ROLE OF ARGININE METHYLATION IN MUSCLE REGENERATION}

Skeletal muscle has a resilient regenerative capacity via stem cells, also called satellite cells. Skeletal muscle regeneration is a multi-step process, including activation of quiescent satellite cells, expansion of activated progenitors, and terminal differentiation and fusion of myoblasts into preexisting myofibers [14,15]. Upon activation, paired box 7 (Pax7)-positive satellite cells express key regulatory factors belonging to the myogenic basic helix-loop-helix (bHLH) family (myogenic differentiation 1 [MyoD], myogenic factor 5 [Myf5], myogenin, and myogenic regulatory factor 4). These factors are critical to regulate myoblast proliferation and differentiation $[16,17]$. MyoD family bHLH factors function as master regulators to induce the expression of elemental muscle-specific genes and myogenic differentiation in non-muscle cells, like fibroblasts $[18,19]$. The activation of MyoD is regulated through multiple mechanisms including heterodimerization of MyoD with its partner E proteins (E12 and E47) that can bind to the consensus DNA sequence called the E-box (CANNTG, where $\mathrm{N}$ is any base), which is found in the regulatory regions of many muscle-specific genes [20-23]. Signaling pathways like p38 mitogen-activated protein kinase (p38MAPK) and AKT promote myogenesis through the activation of MyoD-mediated gene expression via phosphorylation of several transcriptional regulators including E proteins and the chromatin-modifying enzyme switch/sucrose non-fermentable (SWI/ SNF) subunit Brahma-associated factor 60c (BAF60c) [24-28]. Early studies using $\mathrm{C} 2 \mathrm{C} 12$ myoblasts have shown that PRMT4/coactivator-associated arginine methyltransferase 1 (CARM1) and PRMT5 promote myoblast differentiation by regulating myocyte enhancer factor $2 \mathrm{C}$ (Mef2c) or MyoD, respectively $[29,30]$. In myogenesis, MyoD cooperates with histone-modifying enzymes and ATP-dependent chromatin remodeling proteins, such as the Brahma-related gene 1 (BRG1) and BAF subunit of SWI/SNF complexes, to promote the expression of downstream muscle genes [31]. Interestingly, PRMT5 is co-recruited to the myogenin promoter region with BRG1 and MyoD, leading to gene expression [30]. We recently reported that PRMT7 stimulates MyoD-mediated myoblast differentiation through methylation of p38MAPK at arginine residue 70 [32]. Studies with satellite cell-specific deletion mouse models for PRMT1, PRMT4, PRMT5, and PRMT7 have underlined the importance of arginine methylation in muscle stem cell function. PRMT4 regulates Myf5 induction through methylation of Pax7 during asymmetric division of satellite cells, which is critical for activation of satellite cells [33]. PRMT5 regulates muscle stem cell proliferation through silencing the cell cycle inhibitor p21 [34]. PRMT5 deficiency causes reduced H3R8me2s levels at the p21 promoter region, thereby enhancing its expression and leading to reduced proliferation of activated progenitors. PRMT1-deficient satellite cells exhibit enhanced proliferation with defective terminal differentiation through impaired regulation of MyoD [35]. PRMT1 regulates sineoculis homeobox homolog 1 (Six1), a coactivator of MyoD, through arginine methylation, which is critical for MyoD-mediated terminal differentiation. Lastly, it has been shown that PRMT7 controls the regenerative capacity of muscle stem cells in age-related manner. Adult mice null for PRMT7 or with conditional deletion of PRMT7 in satellite cells exhibited significantly impaired muscle regeneration due to premature cell cycle exit and cellular senescence 


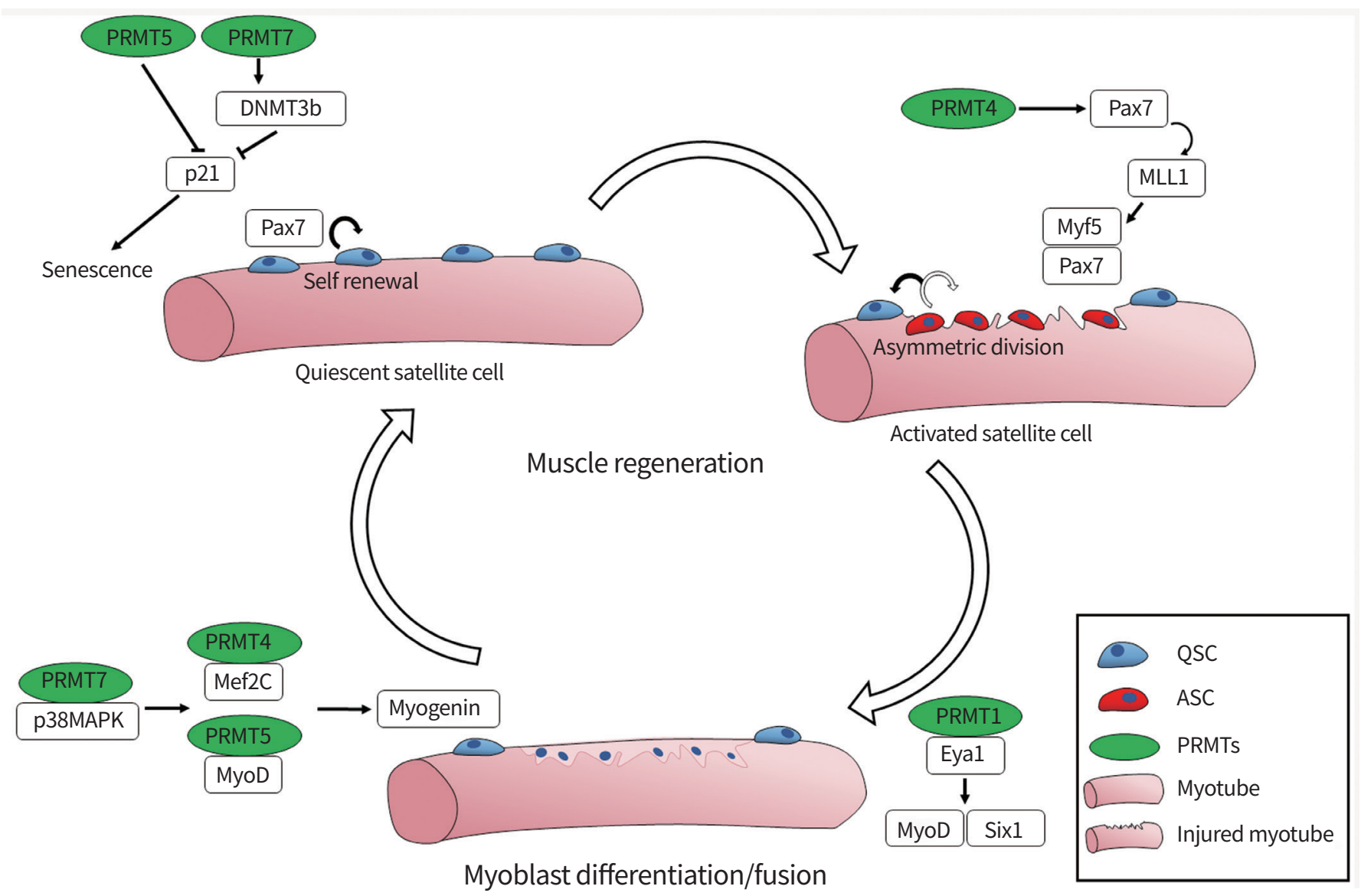

Fig. 1. A scheme summarizing the role of protein arginine methyltransferases (PRMTs) in skeletal muscle regeneration. PRMTs play critical roles in muscle regeneration through modulating muscle-specific gene expression. PRMT5 and 7 play critical roles in preventing the senescence of satellite cells by suppressing the expression of the cell cycle inhibitor p21. Furthermore, PRMT1, 4, 5, and 7 regulate the activities of muscle-specific transcription factors such as paired box 7 (Pax7), myogenic differentiation 1 (MyoD), or myogenin, thereby promoting myogenesis. DNMT3b, DNA methyltransferase 3b; MLL, mixed lineage leukemia; Myf5, myogenic factor 5; p38MAPK, p38 mitogenactivated protein kinase; Mef2C, myocyte enhancer factor 2C; Eya1, EYA transcriptional coactivator and phosphatase 1; Six1, sineoculis homeobox homolog 1; QSC, quiescent satellite cell; ASC, activated satellite cell.

[36]. PRMT7 ablation reduces the level of DNA methyltransferase $3 b$ (DNMT3b), leading to the increased expression of p21 and cellular senescence in activated satellite cells. Based on these studies, it can be concluded that arginine methylation is critical for maintaining muscle stem cell function (Fig. 1). However, few PRMT substrates are known, and the upstream modulators of these enzymes or crosstalk between these enzymes are entirely unknown. Therefore, further studies are required to elucidate the defined mechanisms and function of PRMTs in maintaining muscle regeneration.

\section{THE ROLE OF ARGININE METHYLATION IN SKELETAL MUSCLE METABOLISM}

Recent studies have proposed critical roles of PRMTs in the control of skeletal muscle metabolism. Skeletal muscle ex- hibits a remarkable adaptation capacity in energy metabolism and contractile functions, and responds to various stimuli like exercise, hormones, or nutritional states. Skeletal muscle metabolism is modulated by glucose transport, mitochondrial biogenesis, and protein turnover $[2,5,6,37]$. Skeletal muscle mass is determined by the balanced regulation of protein synthesis and breakdown [38]. Chronic catabolic conditions, such as fasting or denervation, trigger elevated protein degradation and autophagy, thereby leading to muscle wasting. Excessive catabolic pathway signaling is triggered by enhanced AMP-activated protein kinase (AMPK) and p38MAPK activation, accompanied by induction of the proteolytic regulators Forkhead box $\mathrm{O}$ (FoxO), muscle-specific RING finger protein 1 (MuRF1), and atrogin [39-41]. The expression of PRMT1 and 4 is altered in denervation-induced atrophy or muscular dystrophic muscles, suggesting that 
PRECISION AND FUTURE MIEDICINE

Role of Arg methylation in muscle homeostasis

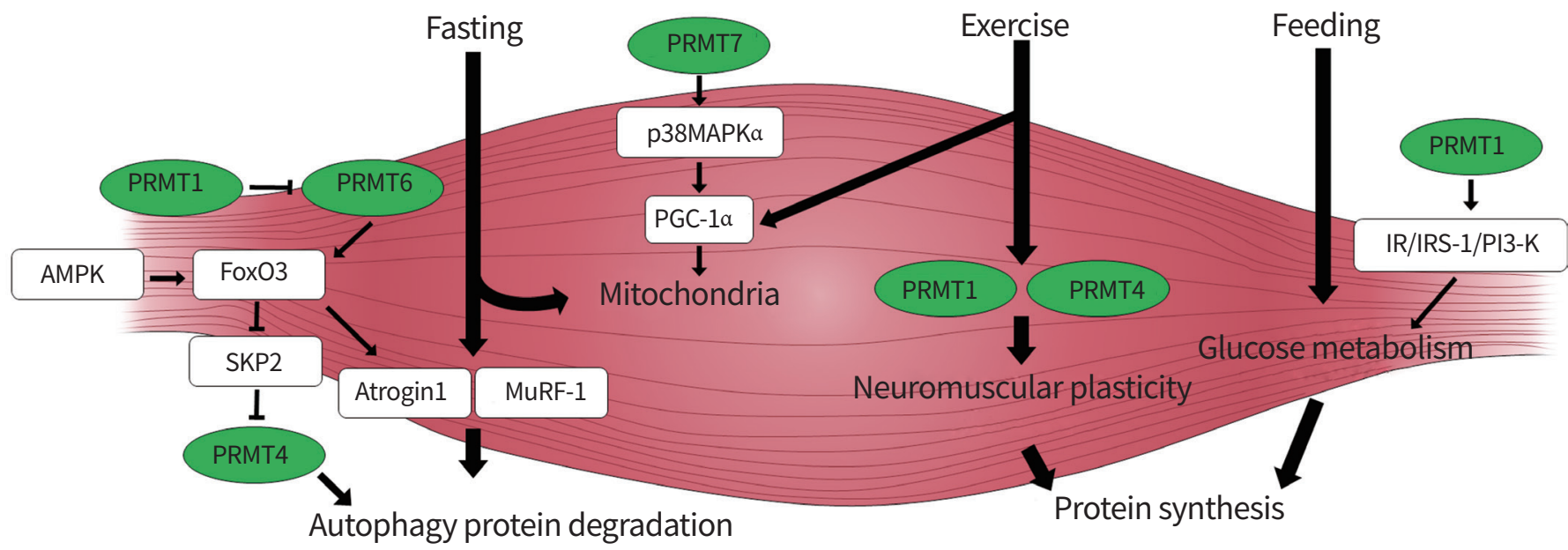

Fig. 2. A model for the role of protein arginine methyltransferases (PRMTs) in the control of skeletal muscle metabolism. PRMTs (green) are implicated in the control of skeletal muscle homeostasis through different mechanisms involving phosphatidylinositol 3-kinase (PI3-K), Forkhead Box 03 (FoxO3), or p38 mitogen-activated protein kinase (p38MAPK) for skeletal muscle homeostasis. AMPK, AMP-activated protein kinase; SKP2, S-phase kinase associated protein 2; MuRF-1, muscle-specific RING finger protein 1; PGC-1a, peroxisome proliferatoractivated receptor gamma coactivator $1 \alpha$; IR, insulin receptor; IRS-1, insulin receptor substrate-1.

these PRMTs might be involved in the atrophy process [42]. Consistently, recent studies have reported that these PRMTs are implicated in controlling the autophagy-proteosomal degradation that is critical for muscle mass control [43]. Muscle-specific ablation of PRMT1 causes muscle atrophy via PRMT6/FoxO3-mediated nuclear accumulation and enhanced autophagy [44]. PRMT4 also contributes to the autophagic process via $\mathrm{FOXO} 3$ methylation in atrophy-induced skeletal muscle [45]. Furthermore, PRMT4-dependent histone arginine methylation is also an essential nuclear event through AMPK-S-phase kinase associated protein 2 (SKP2)-CARM1 for autophagic induction after nutrient starvation [46]. The in vivo function of PRMTs in controlling anabolic pathways is currently unclear. Several in vitro studies on skeletal muscle cells have proposed potential roles of PRMT1 and PRMT4. PRMT1 regulates the insulin receptor (IR)/insulin receptor substrate-1 (IRS-1)/phosphatidylinositol 3-kinase (PI3-K) pathway involved in glucose transport in L6 skeletal muscle cells [47], while PRMT4 is necessary for the expression of genes involved in glycogen metabolism in skeletal muscle cells [48]. Further studies are required to determine the in vivo role of PRMTs in glucose metabolism and anabolic responses of skeletal muscle.

Skeletal muscle remodeling toward an oxidative metabolism is important for muscle function and whole-body metabolism. Recently, PRMT7 has been implicated in muscle oxidative metabolism through activation of p38MAPK/peroxisome proliferator-activated receptor gamma coactivator 1 a (PGC-1a) pathways, contributing to enhanced mitochon- drial biogenesis and function [49]. PRMT7 deficiency causes reduced energy expenditure and induced age-dependent obesity. Previous studies have proposed a role of PRMT1 in inducing mitochondrial genes in non-muscle cells through methylation of p38MAPK and receptor-interacting protein 140 (RIP140) [50,51]. In skeletal muscle, the expression of PRMT1 and PRMT4 is induced by treadmill exercise [52], suggesting potential roles of these PRMTs in enhancing exercise-mediated oxidative metabolism. Further studies are required to define the role of other PRMTs that are expressed in skeletal muscle, the upstream inducers of PRMTs, or the epigenetic regulation of skeletal muscle homeostasis by PRMTs. Regardless of our limited current understanding, PRMTs have emerged as critical regulators in the control of skeletal muscle homeostasis (Fig. 2).

\section{CONCLUSION}

Muscle atrophy is caused by impaired regeneration capacities, metabolic processes, or protein turnover, leading to increased morbidity and mortality. In this review, we present the possibility of new diagnostic markers by understanding the cellular regulatory mechanisms in skeletal muscle maintenance. It is important to know the expression and activity of PRMTs as well as the changes in methylation of the target molecule and the downstream signaling systems in muscle repair and adaptation to stress. Understanding the roles of PRMTs in various steps during muscle cell regeneration and metabolism may provide new potential targets for counter- 
ing muscle weakness and atrophy.

\section{CONFLICTS OF INTEREST}

No potential conflict of interest relevant to this article was reported.

\section{ACKNOWLEDGMENTS}

This work was supported by the grants from the National Research Foundation of Korea Grant funded by the Korean Government (NRF-2019R1I1A1A01062190 to Hyun-Ju Jeong).

\section{ORCID}

Hyebeen Kim https://orcid.org/0000-0002-3066-7098

Jong-Sun Kang https://orcid.org/0000-0001-9072-8211

Hyun-Ju Jeong https://orcid.org/0000-0001-9025-2559

\section{REFERENCES}

1. Reid KF, Fielding RA. Skeletal muscle power: a critical determinant of physical functioning in older adults. Exerc Sport Sci Rev 2012;40:4-12.

2. Brook MS, Wilkinson DJ, Phillips BE, Perez-Schindler J, Philp A, Smith K, et al. Skeletal muscle homeostasis and plasticity in youth and ageing: impact of nutrition and exercise. Acta Physiol (Oxf) 2016;216:15-41.

3. Shulman GI, Rothman DL, Jue T, Stein P, DeFronzo RA, Shulman RG. Quantitation of muscle glycogen synthesis in normal subjects and subjects with non-insulin-dependent diabetes by $13 \mathrm{C}$ nuclear magnetic resonance spectroscopy. N Engl J Med 1990;322:223-8.

4. Zurlo F, Larson K, Bogardus C, Ravussin E. Skeletal muscle metabolism is a major determinant of resting energy expenditure. J Clin Invest 1990;86:1423-7.

5. Rizzoli R, Reginster JY, Arnal JF, Bautmans I, Beaudart C, Bischoff-Ferrari $\mathrm{H}$, et al. Quality of life in sarcopenia and frailty. Calcif Tissue Int 2013;93:101-20.

6. Richter EA, Turcotte L, Hespel P, Kiens B. Metabolic responses to exercise. Effects of endurance training and implications for diabetes. Diabetes Care 1992;15:1767-76.

7. Dutta C. Significance of sarcopenia in the elderly. J Nutr 1997;127(5 Suppl):992S-3S.

8. Gorshunova NK, Medvedev NV. Significance of insulin resistance in the pathogenesis of sarcopenia and chronic heart failure in elderly hypertensive patients. Adv Geron- tol 2016;29:324-28.

9. Wang E, Næss MS, Hoff J, Albert TL, Pham Q, Richardson RS, et al. Exercise-training-induced changes in metabolic capacity with age: the role of central cardiovascular plasticity. Age (Dordr) 2014;36:665-76.

10. Santos AL, Lindner AB. Protein posttranslational modifications: roles in aging and age-related disease. Oxid Med Cell Longev 2017;2017:5716409.

11. Bedford MT, Clarke SG. Protein arginine methylation in mammals: who, what, and why. Mol Cell 2009;33:1-13.

12. Pal S, Sif S. Interplay between chromatin remodelers and protein arginine methyltransferases. J Cell Physiol 2007; 213:306-15.

13. Blanc RS, Richard S. Arginine methylation: the coming of age. Mol Cell 2017;65:8-24.

14. Wang YX, Rudnicki MA. Satellite cells, the engines of muscle repair. Nat Rev Mol Cell Biol 2011;13:127-33.

15. Brack AS, Rando TA. Tissue-specific stem cells: lessons from the skeletal muscle satellite cell. Cell Stem Cell 2012; 10:504-14.

16. Braun T, Gautel M. Transcriptional mechanisms regulating skeletal muscle differentiation, growth and homeostasis. Nat Rev Mol Cell Biol 2011;12:349-61.

17. Sartorelli V, Caretti G. Mechanisms underlying the transcriptional regulation of skeletal myogenesis. Curr Opin Genet Dev 2005;15:528-35.

18. Davis RL, Weintraub H, Lassar AB. Expression of a single transfected cDNA converts fibroblasts to myoblasts. Cell 1987;51:987-1000.

19. Braun T, Buschhausen-Denker G, Bober E, Tannich E, Arnold $\mathrm{HH}$. A novel human muscle factor related to but distinct from MyoD1 induces myogenic conversion in 10T1/2 fibroblasts. EMBO J 1989;8:701-9.

20. Murre C, McCaw PS, Vaessin H, Caudy M, Jan LY, Jan YN, et al. Interactions between heterologous helix-loop-helix proteins generate complexes that bind specifically to a common DNA sequence. Cell 1989;58:537-44.

21. Schwarz JJ, Chakraborty T, Martin J, Zhou JM, Olson EN. The basic region of myogenin cooperates with two transcription activation domains to induce muscle-specific transcription. Mol Cell Biol 1992;12:266-75.

22. Tapscott SJ. The circuitry of a master switch: Myod and the regulation of skeletal muscle gene transcription. Development 2005;132:2685-95.

23. Yang Z, MacQuarrie KL, Analau E, Tyler AE, Dilworth FJ, Cao Y, et al. MyoD and E-protein heterodimers switch rhabdomyosarcoma cells from an arrested myoblast 
phase to a differentiated state. Genes Dev 2009;23:694707.

24. de la Serna IL, Carlson KA, Imbalzano AN. Mammalian SWI/SNF complexes promote MyoD-mediated muscle differentiation. Nat Genet 2001;27:187-90.

25. Simone C, Forcales SV, Hill DA, Imbalzano AN, Latella L, Puri PL. P38 athway targets SWI-SNF chromatin-remodeling complex to muscle-specific loci. Nat Genet 2004;36: 738-43.

26. Lluis F, Ballestar E, Suelves M, Esteller M, Munoz-Canoves P. E47 phosphorylation by $\mathrm{p} 38$ MAPK promotes MyoD/ E47 association and muscle-specific gene transcription. EMBO J 2005;24:974-84.

27. Tran P, Ho SM, Kim BG, Vuong TA, Leem YE, Bae GU, et al. TGF- $\beta$-activated kinase 1 (TAK1) and apoptosis signal-regulating kinase 1 (ASK1) interact with the promyogenic receptor Cdo to promote myogenic differentiation via activation of p38MAPK pathway. J Biol Chem 2012; 287:11602-15.

28. Forcales SV, Albini S, Giordani L, Malecova B, Cignolo L, Chernov A, et al. Signal-dependent incorporation of MyoDBAF60c into Brg1-based SWI/SNF chromatin-remodelling complex. EMBO J 2012;31:301-16.

29. Chen SL, Loffler KA, Chen D, Stallcup MR, Muscat GE. The coactivator-associated arginine methyltransferase is necessary for muscle differentiation: CARM1 coactivates myocyte enhancer factor-2. J Biol Chem 2002;277:432433.

30. Dacwag CS, Ohkawa Y, Pal S, Sif S, Imbalzano AN. The protein arginine methyltransferase Prmt5 is required for myogenesis because it facilitates ATP-dependent chromatin remodeling. Mol Cell Biol 2007;27:384-94.

31. Albini S, Puri PL. SWI/SNF complexes, chromatin remodeling and skeletal myogenesis: it's time to exchange! Exp Cell Res 2010;316:3073-80.

32. Jeong HJ, Lee SJ, Lee HJ, Kim HB, Anh Vuong T, Cho H, et al. Prmt7 promotes myoblast differentiation via methylation of p38MAPK on arginine residue 70. Cell Death Differ 2019 Jun 26 [Epub]. https://doi.org/10.1038/s41418-0190373-y.

33. Kawabe Y, Wang YX, McKinnell IW, Bedford MT, Rudnicki MA. Carm1 regulates Pax7 transcriptional activity through MLL1/2 recruitment during asymmetric satellite stem cell divisions. Cell Stem Cell 2012;11:333-45.

34. Zhang T, Gunther S, Looso M, Kunne C, Kruger M, Kim J, et al. Prmt5 is a regulator of muscle stem cell expansion in adult mice. Nat Commun 2015;6:7140.
35. Blanc RS, Vogel G, Li X, Yu Z, Li S, Richard S. Arginine methylation by PRMT1 regulates muscle stem cell fate. Mol Cell Biol 2017;37:e00457-16.

36. Blanc RS, Vogel G, Chen T, Crist C, Richard S. PRMT7 preserves satellite cell regenerative capacity. Cell Rep 2016; 14:1528-39.

37. Bonaldo P, Sandri M. Cellular and molecular mechanisms of muscle atrophy. Dis Model Mech 2013;6:25-39.

38. Irving BA, Robinson MM, Nair KS. Age effect on myocellular remodeling: response to exercise and nutrition in humans. Ageing Res Rev 2012;11:374-89.

39. Vainshtein A, Grumati P, Sandri M, Bonaldo P. Skeletal muscle, autophagy, and physical activity: the ménage à trois of metabolic regulation in health and disease. J Mol Med (Berl) 2014;92:127-37.

40. Sandri M, Sandri C, Gilbert A, Skurk C, Calabria E, Picard A, et al. Foxo transcription factors induce the atrophy-related ubiquitin ligase atrogin-1 and cause skeletal muscle atrophy. Cell 2004;117:399-412.

41. Sandri M. Autophagy in skeletal muscle. FEBS Lett 2010; 584:1411-6.

42. Stouth DW, Manta A, Ljubicic V. Protein arginine methyltransferase expression, localization, and activity during disuse-induced skeletal muscle plasticity. Am J Physiol Cell Physiol 2018;314:C177-90.

43. Milan G, Romanello V, Pescatore F, Armani A, Paik JH, Frasson $\mathrm{L}$, et al. Regulation of autophagy and the ubiquitin-proteasome system by the FoxO transcriptional network during muscle atrophy. Nat Commun 2015;6:6670.

44. Choi S, Jeong HJ, Kim H, Choi D, Cho SC, Seong JK, et al. Skeletal muscle-specific Prmt1 deletion causes muscle atrophy via deregulation of the PRMT6-FOXO3 axis. Autophagy 2019;15:1069-81.

45. Liu Y, Li J, Shang Y, Guo Y, Li Z. CARM1 contributes to skeletal muscle wasting by mediating $\mathrm{FoxO} 3$ activity and promoting myofiber autophagy. Exp Cell Res 2019;374: 198-209.

46. Shin HJ, Kim H, Oh S, Lee JG, Kee M, Ko HJ, et al. AMPKSKP2-CARM1 signalling cascade in transcriptional regulation of autophagy. Nature 2016;534:553-7.

47. Iwasaki $\mathrm{H}$, Yada T. Protein arginine methylation regulates insulin signaling in L6 skeletal muscle cells. Biochem Biophys Res Commun 2007;364:1015-21.

48. Wang SC, Dowhan DH, Eriksson NA, Muscat GE. CARM1/ PRMT4 is necessary for the glycogen gene expression programme in skeletal muscle cells. Biochem J 2012;444: 323-31. 
49. Jeong HJ, Lee HJ, Vuong TA, Choi KS, Choi D, Koo SH, et al. Prmt7 deficiency causes reduced skeletal muscle oxidative metabolism and age-related obesity. Diabetes 2016;65:1868-82.

50. Mostaqul Huq MD, Gupta P, Tsai NP, White R, Parker MG, Wei LN. Suppression of receptor interacting protein 140 repressive activity by protein arginine methylation. EMBO J 2006;25:5094-104.
51. Teyssier C, Ma H, Emter R, Kralli A, Stallcup MR. Activation of nuclear receptor coactivator PGC-1alpha by arginine methylation. Genes Dev 2005;19:1466-73.

52. Ljubicic V, Khogali S, Renaud JM, Jasmin BJ. Chronic AMPK stimulation attenuates adaptive signaling in dystrophic skeletal muscle. Am J Physiol Cell Physiol 2012; 302:C110-21. 